\title{
Controllability of a Class of Bimodal Discrete-Time Piecewise Linear Systems
}

\author{
E. Yurtseven M.K. Camlibel W.P.M.H. Heemels
}

\begin{abstract}
In this paper we will provide algebraic necessary and sufficient conditions for the controllability/reachability/null controllability of a class of bimodal discrete-time piecewise linear systems including several instances of interest that are not covered by existing works which focus primarily on the planar case. In particular, the class is characterized by a continuous right-hand side, a scalar input and a transfer function from the control input to the switching variable with at most two zeroes whereas the state can be of any dimension. To arrive at the main result, we will make use of geometric control theory for linear systems and a novel result on controllability for inputconstrained linear systems with non-convex constraint sets.

Index Terms-Bimodal systems, piecewise linear systems, controllability, reachability, hybrid systems, non-convex input constraint set
\end{abstract}

\section{INTRODUCTION}

Controllability has always played an important role in modern control theory. Kalman and Hautus studied this notion for linear systems and gave complete characterizations in algebraic forms. In the presence of input constraints, characterizations for the controllability of discrete-time linear systems were given in [1], [2], [3]. In case of hybrid dynamical systems, such as piecewise linear systems, such complete characterizations of null controllability, reachability or controllability are hard to come by. Indeed, it is known from [4] that certain controllability problems for discrete-time piecewise linear systems in a general setting are undecidable. However, several results were obtained on the controllability of different subclasses of piecewise linear systems. In [5], [6], $\mathrm{Xu}$ and Xie give characterizations for the controllability of discrete-time planar bimodal piecewise linear systems. Brogliato obtains necessary and sufficient conditions for the controllability of a class of continuous-time piecewise linear systems in [7], but his results only apply to planar systems as well. Bemporad et al. [8] propose an algorithmic approach based on optimization tools. Although this approach makes it possible to check controllability of a given discrete-time

Evren Yurtseven is with the Eindhoven University of Technology, Dept. of Mechanical Engineering, Hybrid and Networked Systems group, The Netherlands and SKF, Industrial Division, System and Product Development, The Netherlands evren.yurtseven@skf.com, evren.yurtseven@gmail.com

Kanat Camlibel is with the University of Groningen, Inst. of Mathematics and Computer Science, Groningen, The Netherlands and Dogus University, Dept. of Electronics and Communication Engineering, Turkey m.k.camlibel@ rug.nl, kcamlibel@dogus.edu.tr

Maurice Heemels is with the Eindhoven University of Technology, Dept. of Mechanical Engineering, Hybrid and Networked Systems group, The Netherlands m.heemels@tue.nl

This work was partially supported by the European Commission through project MOBY-DIC Model-based synthesis of digital electronic circuits for embedded control (FP7-INFSO-ICT-248858), http://www.mobydic-project.eu. system, it does not allow drawing conclusions about any general class of systems. Arapostathis and Broucke give a fairly complete treatment of stability and controllability of continuous-time planar conewise linear systems in [9], which are piecewise linear systems for which the regions are convex cones. In [10], Lee and Arapostathis provide a characterization of controllability for a class of continuoustime piecewise linear systems but they assume, among other things, that the number of inputs in each subsystem is one less than the number of states. In [11], Camlibel et al. give algebraic necessary and sufficient conditions for the controllability of a class of continuous-time conewise linear systems, while stabilizability characterizations for bimodal piecewise linear systems are given in [12].

In this paper we present algebraic necessary and sufficient conditions for the controllability, reachability and null controllability of a class of bimodal discrete-time piecewise linear systems. The class is characterized by the property that the dynamics is continuous across the switching plane and that the input is scalar. We impose no restriction on the dimension of the state variable as opposed to the earlier mentioned works [9], [6], [5], [7] that study the controllability of planar piecewise linear systems. Also, we do not adopt the assumption in [10], namely that the number of inputs is one less than the number of states. However, we assume that the transfer function from the control input to the switching variable, i.e. the variable that determines the active mode, has at most two zeroes. This seemingly rather odd assumption forms a complexity boundary in solving this problem for larger classes of PWL systems. Allowing three or more zeroes in the transfer function necessitates solving the controllability problem for discrete-time linear systems with non-convex input constraint sets, which is known to be a very hard problem. In spite of this assumption, our results are the first to provide algebraic necessary and sufficient conditions for the controllability for the indicated class of piecewise linear systems that contains several instances of interest not covered by the existing results. At the end of the paper we will demonstrate this fact with an example whose controllability could indeed not have been checked by earlier works.

The paper is organized as follows. We lay the groundwork to solve the controllability problem in Section II and we define the class of systems we are interested in Section III. In Section IV, V and VI we consider two different problems whose solutions are needed to tackle the main problem. In Section VII we present our main results. Conclusions are presented in section VIII. The proofs of all the results including 
the main result can be found in [13] unless otherwise stated. For space reasons they are omitted in this paper.

\section{DEFINITIONS}

\section{A. Definitions}

Consider the discrete-time system

$$
x[k+1]=g(x[k], u[k]) \quad y[k]=h(x[k])
$$

where $x[k] \in \mathbb{R}^{n}, u[k] \in \mathbb{R}^{m}, y[k] \in \mathbb{R}^{p}$ are the state, the input and the output variable, respectively, at discrete time $k \in \mathbb{N}$. Here, $g: \mathbb{R}^{n} \times \mathbb{R}^{m} \rightarrow \mathbb{R}^{n}$ and $h: \mathbb{R}^{n} \rightarrow$ $\mathbb{R}^{p}$ are given functions. Given an initial state $x_{0} \in \mathbb{R}^{n}$ and an input sequence $\mathbf{u}=\{u[0], \ldots, u[N]\}$ with $N \in \mathbb{N}$, we will denote the state trajectory corresponding to $\mathbf{u}$ and the initial state $x[0]=x_{0}$ by $x_{x_{0}, \mathbf{u}}$. Likewise, we will denote the corresponding output sequence by $y_{x_{0}, \mathbf{u}}$.

Definition II.1 Consider the system (1). Let $\mathbf{y}=\{y[0]$, $\ldots, y[N]\}$ be a sequence with $y[k] \in \mathbb{R}^{p}, k=0, \ldots, N$. Given an initial state $x_{0} \in \mathbb{R}^{n}$, we say that $x_{0}$ is compatible with $\mathrm{y}$, if there exists an input sequence $\mathbf{u}=\{u[0], \ldots, u[N-1]\}$ such that $y_{x_{0}, \mathbf{u}}[k]=y[k]$ for $k=0, \ldots, N$. Likewise, given an input sequence $\overline{\mathbf{u}}=$ $\{\bar{u}[0], \ldots, \bar{u}[N-1]\}$, we say that $\overline{\mathbf{u}}$ is compatible with $\mathbf{y}$, if there exists an initial state $\bar{x}_{0} \in \mathbb{R}^{n}$ such that $y_{\bar{x}_{0}, \overline{\mathbf{u}}}[k]=y[k]$ for $k=0, \ldots, N$.

Definition II.2 Consider the system (1). We say that (1) is

- null controllable if for all $x_{0} \in \mathbb{R}^{n}$ there exist an $N \in \mathbb{N}$ and an input sequence $\mathbf{u}=\{u[0], \ldots, u[N-1]\}$ such that $x_{x_{0}, \mathbf{u}}[N]=0$.

- reachable if for all $x_{f} \in \mathbb{R}^{n}$ there exist an $N \in \mathbb{N}$ and an input sequence $\mathbf{u}=\{u[0], \ldots, u[N-1]\}$ such that $x_{0, \mathbf{u}}[N]=x_{f}$.

- controllable iffor all $x_{0}, x_{f} \in \mathbb{R}^{n}$ there exist an $N \in \mathbb{N}$ and an input sequence $\mathbf{u}=\{u[0], \ldots, u[N-1]\}$ such that $x_{x_{0}, \mathbf{u}}[N]=x_{f}$.

\section{B. Classical Results}

Consider the linear system

$$
x[k+1]=A x[k]+B u[k]
$$

with $x[k] \in \mathbb{R}^{n}$ being the state and $u[k] \in \mathbb{R}^{m}$ being the input, together with the input constraint

$$
u[k] \in \mathcal{U}, \quad k \in \mathbb{N}
$$

where $\mathcal{U} \subseteq \mathbb{R}^{m}$ is a solid polyhedral closed cone, i.e. there exists a matrix $U \in \mathbb{R}^{l \times m}$ for some $l \in \mathbb{N}$ such that $\mathcal{U}=\left\{u \in \mathbb{R}^{m} \mid U u \geq 0\right\}$ and $\mathcal{U}$ has a nonempty interior. The inequalities in $U u \geq 0$ are to be interpreted componentwise. The definitions of null controllability, reachability and controllability as in Definition II.2 are similar for the constrained system (2)-(3) with the understanding that (3) should hold for the input sequence.
Definition II.3 Let $\mathcal{U} \subseteq \mathbb{R}^{m}$ be a nonempty set. We define the dual cone $\mathcal{U}^{*}$ of $\mathcal{U}$ as

$$
\mathcal{U}^{*}:=\left\{v \in \mathbb{R}^{m} \mid v^{\top} u \geq 0 \quad \forall u \in \mathcal{U}\right\}
$$

The following lemma can be based on [14], [1], [2].

Lemma II.4 Let $\mathcal{U}$ be a solid polyhedral closed cone. The constrained system (2)-(3) is

- null controllable if, and only if, the following implications hold:

$$
\begin{aligned}
& \left.\begin{array}{l}
\lambda \in \mathbb{C} \backslash\{0\}, \quad z \in \mathbb{C}^{n}, \\
z^{\top} A=\lambda z^{\top}, z^{\top} B=0
\end{array}\right\} \Rightarrow z=0 \\
& \left.\begin{array}{l}
\lambda \in(0, \infty), z \in \mathbb{R}^{n}, \\
z^{\top} A=\lambda z^{\top}, B^{\top} z \in \mathcal{U}^{*}
\end{array}\right\} \Rightarrow z=0
\end{aligned}
$$

- reachable/controllable if, and only if, the following implications hold:

$$
\begin{aligned}
& \left.\begin{array}{l}
\lambda \in \mathbb{C}, z \in \mathbb{C}^{n}, \\
z^{\top} A=\lambda z^{\top}, z^{\top} B=0
\end{array}\right\} \Rightarrow z=0 \\
& \left.\begin{array}{l}
\lambda \in[0, \infty), z \in \mathbb{R}^{n}, \\
z^{\top} A=\lambda z^{\top}, B^{\top} z \in \mathcal{U}^{*}
\end{array}\right\} \Rightarrow z=0
\end{aligned}
$$

\section{PRoblem Definition}

In this paper we are interested in the null controllability/reachability/controllability of bimodal piecewise linear (PWL) systems that are of the form

$$
\begin{gathered}
x[k+1]= \begin{cases}A_{1} x[k]+B_{1} u[k] & y[k] \geq 0 \\
A_{2} x[k]+B_{2} u[k] & y[k] \leq 0\end{cases} \\
y[k]=C^{\top} x[k]
\end{gathered}
$$

where $x[k] \in \mathbb{R}^{n}$ is the state, $u[k] \in \mathbb{R}$ is the scalar input, $y[k] \in \mathbb{R}$ is a variable determining the active mode at discrete time $k \in \mathbb{N}$ and the matrices $A_{1}, A_{2} \in \mathbb{R}^{n \times n}, B_{1}, B_{2}, C \in$ $\mathbb{R}^{n}$ are given.

We assume $C \neq 0$ and that the right-hand side of (6) is continuous. This is equivalent to the existence of a vector $E \in \mathbb{R}^{n}$ such that

$$
A_{2}=A_{1}+E C^{\top} \text { and } B_{1}=B_{2}=: B
$$

To see why (7) holds, we express the continuity assumption as follows.

$$
\left[\begin{array}{ll}
C^{\top} & 0
\end{array}\right]\left[\begin{array}{l}
x \\
u
\end{array}\right]=0 \Rightarrow\left[\begin{array}{ll}
A_{1}-A_{2} & B_{1}-B_{2}
\end{array}\right]\left[\begin{array}{l}
x \\
u
\end{array}\right]=0
$$

Stated otherwise,

$$
\operatorname{ker}\left(\left[\begin{array}{ll}
C^{\top} & 0
\end{array}\right]\right) \subseteq \operatorname{ker}\left(\left[\begin{array}{ll}
A_{1}-A_{2} & B_{1}-B_{2}
\end{array}\right]\right)
$$

Then, it is possible to show that for some vector $E \in \mathbb{R}^{n}$

$$
\left[\begin{array}{ll}
A_{1}-A_{2} & B_{1}-B_{2}
\end{array}\right]^{\top}=\left[\begin{array}{ll}
C^{\top} & 0
\end{array}\right]^{\top} E^{\top}
$$

To show that this controllability problem is far from being trivial and that the controllability of (6) cannot be inferred from the controllability properties of the subsystems only, we would like to give a motivating example. 
Example III.1 Consider the PWL system given by

$$
\begin{aligned}
x_{1}[k+1]= & \begin{cases}x_{1}[k]-x_{2}[k] & x_{2}[k] \geq 0 \\
x_{1}[k]+x_{2}[k] & x_{2}[k] \leq 0\end{cases} \\
& x_{2}[k+1]=u[k]
\end{aligned}
$$

In this example, both of the subsystems are controllable as linear systems, as is easily verified. However, the piecewise linear system is not controllable as $x_{1}[k] \leq x_{1}[0], k \in \mathbb{N}$. This shows that controllability of a PWL system cannot be characterized only in terms of the controllability of its subsystems.

Define the transfer functions $G_{i}(z)=C^{\top}\left(z I-A_{i}\right)^{-1} B$ for $i=1,2$. It follows from (7) that $\sigma$ is a zero of $G_{1}(z)$ if and only if it is a zero of $G_{2}(z)$ and that $G_{1}(z) \equiv 0$ if and only if $G_{2}(z) \equiv 0$. In the rest of the paper we will assume that $G_{1}(z) \not \equiv 0$, as otherwise the system (6) would not be controllable, and that it has at most two zeroes. We will provide insights on the need of this rather odd requirement later. Let $\mathcal{V}_{i}^{*}$ be the largest $\left(A_{i}, B\right)$-invariant subspace contained in $\operatorname{ker} C^{\top}$ for $i=1,2$, i.e. $\mathcal{V}_{i}^{*}$ is the largest of the subspaces, $\mathcal{V}_{i}$, that satisfy $\left(A_{i}+B F_{i}\right) \mathcal{V}_{i} \subseteq$ $\mathcal{V}_{i} \subseteq \operatorname{ker} C^{\top}$ for some $F_{i} \in \mathbb{R}^{1 \times n}, i=1,2$. We will denote the set $\left\{F_{i} \in \mathbb{R}^{1 \times n} \mid\left(A_{i}+B F_{i}\right) \mathcal{V}_{i}^{*} \subseteq \mathcal{V}_{i}^{*} \subseteq \operatorname{ker} C^{\top}\right\}$ by $\mathcal{F}_{i}^{*}$. Likewise, let $\mathcal{Z}_{i}^{*}$ be the smallest $\left(C^{\top}, A_{i}\right)$-invariant subspace that contains im $B$ for $i=1,2$, i.e. $\mathcal{Z}_{i}^{*}$ is the smallest of the subspaces, $\mathcal{Z}_{i}$, that satisfy $\left(A_{i}+K_{i} C^{\top}\right) \mathcal{Z}_{i} \subseteq \mathcal{Z}_{i}$ and im $B \subseteq$ $\mathcal{Z}_{i}$ for some $K_{i} \in \mathbb{R}^{n \times 1}, i=1,2$. See [15], [16], [17] for a detailed discussion on these particular subspaces. Since $G_{i}(z) \not \equiv 0, i=1,2, G_{i}(z)$ is invertible as a rational function. Therefore, $\mathbb{R}^{n}$ admits the decomposition $\mathbb{R}^{n}=\mathcal{V}_{i}^{*} \oplus \mathcal{Z}_{i}^{*}$ for $i=1,2$. See [15] for the proof of this implication. Due to (7), it holds that $\mathcal{V}_{1}^{*}=\mathcal{V}_{2}^{*}=: \mathcal{V}^{*}, \mathcal{Z}_{1}^{*}=\mathcal{Z}_{2}^{*}=: \mathcal{Z}^{*}$ and $\mathcal{F}_{1}^{*}=\mathcal{F}_{2}^{*}=: \mathcal{F}^{*}$. First, we apply the pre-compensating state feedback $u[k]=F x[k]+v[k]$ to (6) with $F \in \mathcal{F}^{*}$. Due to (7) we have that $\left.\left(A_{1}+B F\right)\right|_{\mathcal{V}^{*}}=\left.\left(A_{2}+B F\right)\right|_{\mathcal{V} *}$ Then, we apply the similarity transformation, $\tilde{x}=T^{-1} x$, with $T=\left[\begin{array}{ll}T_{1} & T_{2}\end{array}\right]$ where im $T_{1}=\mathcal{V}^{*}$ and im $T_{2}=\mathcal{Z}^{*}$. For ease of exposition, we will not use a different symbol for the new state vector and we will denote $v[k]$ by $u[k]$. Then, we obtain the following representation of (6) that is easier to deal with for characterizing null controllability, reachability and controllability:

$$
\begin{aligned}
x_{1}[k+1] & =H x_{1}[k]+ \begin{cases}g_{1} y[k] & y[k] \geq 0 \\
g_{2} y[k] & y[k] \leq 0\end{cases} \\
x_{2}[k+1] & = \begin{cases}J_{1} x_{2}[k]+b u[k] & y[k] \geq 0 \\
J_{2} x_{2}[k]+b u[k] & y[k] \leq 0\end{cases} \\
y[k] & =c^{\top} x_{2}[k]
\end{aligned}
$$

in which $x_{1}[k], g_{1}, g_{2}, f \in \mathbb{R}^{n_{1}}, x_{2}[k], b, c \in \mathbb{R}^{n_{2}}$ for $k \in \mathbb{N}$ and $H \in \mathbb{R}^{n_{1} \times n_{1}}, J_{1}, J_{2} \in \mathbb{R}^{n_{2} \times n_{2}}$ where $n_{1}=\operatorname{dim} \mathcal{V}^{*}$, $n_{2}=\operatorname{dim} \mathcal{Z}^{*}$ and $n_{1}+n_{2}=n$. Due to the assumption we made on the number of zeroes of $G_{1}(z)$, we have that $0 \leq n_{1} \leq 2$.
Obviously, controllability properties are invariant under pre-compensating feedbacks and similarity transformations. Hence null controllability/reachability/controllability of (9) is equivalent to null controllability/reachability/ controllability of (6). We will now study particular properties of the subsystems (9a) and (9b) that are useful for the main developments. The subsystem (9b) belongs to a special class of systems which we will introduce and analyze in the next section. In section VI we will analyze the subsystem (9a), which we call a push-pull type of system. Note that $\tilde{G}_{i}(z)=c^{\top}\left(z I-J_{i}\right)^{-1} b \not \equiv 0$ for $i=1,2$. Let $\tilde{\mathcal{V}}_{i}^{*}$ denote the largest $\left(J_{i}, b\right)$-invariant subspace contained in $\operatorname{ker} c^{\top}$ for $i=1,2$. Then, $\tilde{\mathcal{V}}_{1}^{*}=\tilde{\mathcal{V}}_{2}^{*}=\{0\}$. Let $\tilde{\mathcal{Z}}_{i}^{*}$ denote the smallest $\left(c^{\top}, J_{i}\right)$-invariant subspace that contains $\operatorname{im} b$. Then, $\tilde{\mathcal{Z}}_{1}^{*}=$ $\tilde{\mathcal{Z}}_{2}^{*}=\mathbb{R}^{n_{2}}$. Due to (7), we also have that $J_{2}=J_{1}+e_{2} c^{\top}$ and

$$
g_{2}=g_{1}+e_{1}
$$

where $\left(\begin{array}{l}e_{1} \\ e_{2}\end{array}\right)=T^{-1} E$ and $C^{\top} T=\left[\begin{array}{ll}0 & c^{\top}\end{array}\right]$.

IV. ObSERVABILITy AND InVERTIBILITy of a SPeCial Class of Bimodal DisCRETE-TIME PIECEWise LiNeAR SYSTEMS

Consider the discrete-time piecewise linear system

$$
\begin{gathered}
x[k+1]=\left\{\begin{array}{cc}
A_{1} x[k]+B u[k] & y[k] \geq 0 \\
A_{2} x[k]+B u[k] & y[k] \leq 0
\end{array}\right. \\
y[k]=C^{\top} x[k]
\end{gathered}
$$

where $x[k] \in \mathbb{R}^{n}, u[k], y[k] \in \mathbb{R}$ for $k \in \mathbb{N}$ and $A_{1}, A_{2} \in$ $\mathbb{R}^{n \times n}, B, C \in \mathbb{R}^{n \times 1}$. We will study a special class of the system (11) as we will need these results later in proving our main result. In particular, we adopt the following assumptions.

Assumption IV.1 The following statements hold.

1) The transfer functions $G_{i}(z)=C^{\top}\left(z I-A_{i}\right)^{-1} B \not \equiv 0$ for $i=1,2$;

2) The right-hand side of (11a) is continuous, i.e. $A_{2}=$ $A_{1}+E C^{\top}$ for some vector $E \in \mathbb{R}^{n}$;

3) The largest $\left(A_{i}, B\right)$-invariant subspace contained in $\operatorname{ker} C^{\top}, \mathcal{V}_{i}^{*}$, is $\{0\}$ for $i=1,2$.

4) The smallest $\left(C^{\top}, A_{i}\right)$-invariant subspace containing $\operatorname{im} B, \mathcal{Z}_{i}^{*}$, is $\mathbb{R}^{n}$ for $i=1,2$.

We would like to point out that the system (9b) belongs to this special class of discrete-time piecewise linear systems.

Corollary IV.2 Consider the system (11) and suppose Assumption IV.1 holds. Then, the following statements hold.

1) $\left(C^{\top}, A_{i}\right)$ is observable,

2) $\left(A_{i}, B\right)$ is controllable,

3) $C^{\top} B=C^{\top} A_{i} B=\ldots=C^{\top} A_{i}^{n-2} B=0$,

4) $C^{\top} A_{i}^{n-1} B \neq 0$

for $i=1,2$. 
Definition IV.3 We say that (11) is both observable and invertible if for any output sequence $\mathbf{y}=\{y[0], y[1], \ldots\}$ of infinite length there exist a unique initial state $x_{0} \in \mathbb{R}^{n}$ and a unique input sequence of infinite length, $\{u[0], u[1], \ldots\}$, that are both compatible with $\mathbf{y}$.

Proposition IV.4 Consider the system (11) for which Assumption IV.1 holds. Then, it is both observable and invertible. In particular, the first $n$ entries of the output sequence $\{y[0], \ldots, y[n-1]\}$ uniquely determine the initial state $x_{0}$.

\section{INSIGHTS ON THE COMPLEXITy OF THE CONTROLLABILITY PROBLEM}

Lemma V.1 The system (6) for which (7) holds with $G_{i}(z)$ $=C^{\top}\left(z I-A_{i}\right)^{-1} B \not \equiv 0, i=1,2$, is controllable if and only if the push-pull system (9a) is controllable.

Corollary V.2 The system (6) for which (7) holds with $G_{i}(z)=C^{\top}\left(z I-A_{i}\right)^{-1} B \not \equiv 0, i=1,2$, is null controllable if and only if the push-pull system (9a) is null controllable.

Now that we have established the equivalency of the controllabilities of (6) and (9a), we embark upon the task of characterizing the controllability of the push-pull system (9a). Although we will give the results on the controllability of push-pull systems in the next section, first we would like to discuss the complexities inherent in this problem. We start with the framework under which we will discuss the controllability of push-pull systems.

A bimodal discrete-time push-pull system with a scalar input is given by

$$
x[k+1]=A x[k]+ \begin{cases}B_{+} u[k] & u[k] \geq 0 \\ B_{-} u[k] & u[k] \leq 0\end{cases}
$$

where $x[k] \in \mathbb{R}^{n}, u[k] \in \mathbb{R}$ for $k \in \mathbb{N}$ and $A \in \mathbb{R}^{n \times n}$, $B_{+}, B_{-} \in \mathbb{R}^{n}$. Note that (9a) fits in this framework with $y$ considered as the "input".

The system (12) can be written as the following equivalent input-constrained linear system.

$$
x[k+1]=A x[k]+\left[B_{+}-B_{-}\right] v[k] \quad \text { with } \quad v[k] \in \tilde{\mathcal{U}}
$$

where $\tilde{\mathcal{U}}=[0, \infty) \times\{0\} \bigcup\{0\} \times[0, \infty) \cdot \tilde{\mathcal{U}}$ is clearly a nonconvex set. Therefore, solving the controllability problem for the PWL system (6) is equivalent to solving the controllability problem for the discrete-time linear system (13) with a particular non-convex input constraint set. Even though controllability of continuous-time linear systems with nonconvex input constraint sets can be solved by convexification of the input constraint set through incorporating all the Filippov solutions (see e.g. [18], [11]), clearly this cannot be done for discrete-time systems.

In the next section we will give the controllability characterization for (13) under the state dimension restriction $n \in$ $\{1,2\}$. Solving the controllability problem for (13) without imposing any restriction on the dimension of the state would allow us to remove the assumption we made on the maximum number of zeroes of $G_{1}(z)$ earlier in Section III. However, the proof of our results on the controllability of (13) (see [13]) is based on a case-by-case analysis where each case corresponds to a different Jordan form of the matrix $A$ and at higher dimensions, $n \geq 3$, such a case-by-case analysis is a very complicated, if not impossible, task. Thus, a structural approach in characterizing controllability of (13) is indispensable if one wants to solve the controllability problem for (6) in a broader generality, that is without limitation on the maximum number of zeroes of $G_{1}(z)$ as long as the number of zeroes is finite. But, to the best of our knowledge, controllability of discrete-time linear systems with non-convex input constraint sets has not been solved so far and it is known to be a very hard problem. That is why in this paper we only deal with systems of the form (6) having at most two zeroes. Despite this, our results are still the first in characterizing controllability for the class of systems described in Section III and this class is not captured by any of the works in the literature.

Now we are ready to state our results on the controllability of push-pull systems of the form (12) for which $n=1,2$ holds. We would like to stress that these results are novel findings on the controllability of discrete-time linear systems with non-convex input constraint sets.

\section{Controllability of Bimodal Discrete-Time PUSH-PULL SYSTEMS}

Theorem VI.1 Consider the system (12) with $n \in\{1,2\}$. The following statements are equivalent.

(i) (12) is reachable.

(ii) The linear system given by

$$
x[k+1]=A x[k]+\left[B_{+}-B_{-}\right] v[k] \text { with } v[k] \in \overline{\mathcal{U}}
$$

where $\overline{\mathcal{U}}=\mathbb{R}_{+}^{2}$ is reachable.

Corollary VI.2 Consider the system (12) with $n \in\{1,2\}$. The following statements are equivalent.

(i) The system (12) is null controllable.

(ii) The system (14) is null controllable with the input constraint $v[k] \in \overline{\mathcal{U}}:=\mathbb{R}_{+}^{2}$.

Corollary VI.3 Consider the system (12) with $n \in\{1,2\}$. The following statements are equivalent.

(i) The system (12) is controllable.

(ii) The system (14) is controllable with the input constraint $v[k] \in \overline{\mathcal{U}}:=\mathbb{R}_{+}^{2}$.

\section{MAIN RESUlts}

We are now in a position to present the main result of this paper, which follows from combining Lemmas II.4, V.1 and Corollaries V.2, VI.2 and VI.3.

Theorem VII.1 Consider the system (6) for which (7) holds with $G_{i}(z)=C^{\top}\left(z I-A_{i}\right)^{-1} B \not \equiv 0$ and $G_{i}(z)$ having at most two zeroes, $i=1,2$. Then the following statements hold. 
1) The system (6) is null controllable if and only if the following implications hold:

$$
\left.\begin{array}{c}
\left.\lambda \in \mathbb{C} \backslash\{0\}, z \in \mathbb{C}^{n}, \quad i=1,2\right\} \Rightarrow z=0 \\
z^{\top} A_{i}=\lambda z^{\top}, z^{\top} B=0 \\
\lambda \in(0, \infty), z \in \mathbb{R}^{n}, \\
w_{1} \leq 0, w_{2} \geq 0 \\
{\left[\begin{array}{ll}
z^{\top} & w_{i}
\end{array}\right]\left[\begin{array}{cc}
A_{i}-\lambda I & B \\
C^{\top} & 0
\end{array}\right]=0}
\end{array} \quad i=1,2\right\} \Rightarrow z=0
$$

2) The system (6) is controllable if and only if the following implications hold.

$$
\begin{gathered}
\left.\begin{array}{l}
\lambda \in \mathbb{C}, z \in \mathbb{C}^{n}, \\
z^{\top} A_{i}=\lambda z^{\top}, z^{\top} B=0 \quad i=1,2
\end{array}\right\} \Rightarrow z=0 \\
\lambda \in[0, \infty), z \in \mathbb{R}^{n}, \\
w_{1} \leq 0, w_{2} \geq 0 \\
{\left[\begin{array}{ll}
z^{\top} & w_{i}
\end{array}\right]\left[\begin{array}{cc}
A_{i}-\lambda I & B \\
C^{\top} & 0
\end{array}\right]=0}
\end{gathered}
$$

Remark VII.2 Before we conclude the paper, we would like to give an example of a bimodal discrete-time piecewise linear system whose controllability could not have been checked by previous works but only can be checked by the new results in our paper. The PWL system (6) given by the matrices

$$
\begin{gathered}
A_{1}=\left[\begin{array}{ccc}
-1 & 0 & 0 \\
0 & 0 & 0 \\
0 & 0 & 1
\end{array}\right], A_{2}=\left[\begin{array}{ccc}
0 & -2 & 1 \\
0 & 0 & 0 \\
0 & 0 & 1
\end{array}\right] \\
B_{1}=B_{2}=\left[\begin{array}{l}
1 \\
1 \\
1
\end{array}\right], C=\left[\begin{array}{c}
1 \\
-2 \\
1
\end{array}\right]
\end{gathered}
$$

This system has three states and one input, thus it does not fall under the framework of the results in [5], [10]. Note that the transfer functions $G_{i}(z)=C^{\top}\left(z I-A_{i}\right)^{-1} B, i=1,2$, have no zeroes, which means that this PWL system is covered by the results in this paper. Indeed, using Theorem VII.1, it is easily verified that this PWL system is controllable.

Now, we will show with hand calculation that this system is controllable. First, we take $x[0]=0$ and we want to steer the state to some arbitrary $x_{f}$ in finite time, say $r \in \mathbb{N}$, i.e. $x[r]=x_{f}$. It is easy to see that $\left.x[1]=\left[\begin{array}{ll}u[0] & u[0] \\ u[0\end{array}\right]\right]^{\top}$. As $y[1] \equiv 0, x[2]$ is deterministically given as $x[2]=$ $[-u[0]+u[1] \quad u[1] \quad u[0]+u[1]]^{\top}$. Since $y[2] \equiv 0$, we have

$$
x[3]=[u[0]-u[1]+u[2] \quad u[2] \quad u[0]+u[1]+u[2]]^{\top}
$$

We can write this last equality as

$$
\left[\begin{array}{ccc}
1 & -1 & 1 \\
0 & 0 & 1 \\
1 & 1 & 1
\end{array}\right]\left[\begin{array}{l}
u[0] \\
u[1] \\
u[2]
\end{array}\right]=x[3]
$$

Due to the invertibility of the $3 \times 3$ matrix in the above equation, for any given $x[3]=x_{f}$ one can find the appropriate input sequence $\{u[0], u[1], u[2]\}$ to steer the state of the system from the origin to $x_{f}$. Thus, we have established that this PWL system is reachable.

By a similar reasoning and hand calculation, one can also show null controllability of this system. Since this system is reachable and null controllable, we conclude that it is controllable.

\section{CONCLUSION}

In this paper we presented algebraic characterizations of the controllability, reachability and null controllability of a class of discrete-time bimodal piecewise linear systems. By using geometric control theory we showed that the controllability problem is equivalent to the controllability of a particular subsystem of the original system, which is in the form of a so-called push-pull system. By studying the controllability of linear systems with a particular non-convex input constraint set, we derived conditions for the controllability of push-pull systems up to a certain dimension, hence leading to our main result.

\section{REFERENCES}

[1] M.E. Evans, "The convex controller: controllability in finite time," International Journal of Systems Science, vol. 16, no. 1, pp. 31-47, 1985.

[2] K.S. Nguyen, "On the null-controllability of linear discrete-time systems with restrained controls," J. Optimization Theory Appl., vol. 50, no. 2, pp. 313-329, 1986.

[3] M.E. Evans and D.N.P. Murthy, "Controllability of discrete-time systems with positive controls," IEEE Trans. Automat. Control, vol. 22, no. 6, pp. 942-945, 1977.

[4] V.D. Blondel and J.N. Tsitsiklis, "Complexity of stability and controllability of elementary hybrid systems," Automatica, vol. 35, no. 3, pp. 479-490, 1999

[5] J. Xu and L. Xie, "Controllability and reachability of discrete-time planar bimodal piecewise linear systems," in Proc. of the American Control Conference, 2006, pp. 4387-4392.

[6] — , "Null controllability of discrete-time planar bimodal piecewise linear systems," International Journal of Control, vol. 78, no. 18, pp. 1486-1496, 2005.

[7] B. Brogliato, "Some results on the controllability of planar variational inequalities," Systems and Control Letters, vol. 55, no. 1, pp. 65-71, 2005.

[8] A. Bemporad, G. Ferrari-Trecate, and M. Morari, "Observability and controllability of piecewise affine and hybrid systems," IEEE Trans. Automat. Control, vol. 45, no. 10, pp. 1864-1876, 2000.

[9] A. Arapostathis and M.E. Broucke, "Stability and controllability of planar, conewise linear systems," Syst. Control Lett., vol. 56, no. 12, pp. 150-158, 2007.

[10] K.K. Lee and A. Arapostathis, "On the controllability of piecewise linear hypersurface systems," Syst. Control Lett., vol. 55, no. 1, pp. 65-71, 1987.

[11] M.K. Camlibel, W.P.M.H. Heemels, and J.M. Schumacher, "Algebraic necessary and sufficient conditions for the controllability of conewise linear systems," IEEE Transactions on Automatic Control, vol. 53, no. 3, pp. 762-774, 2008.

[12] — - "Stabilizability of bimodal piecewise linear systems with continuous vector field," Automatica, vol. 44, no. 5, pp. 1261-1267, 2008.

[13] E. Yurtseven, M.K. Camlibel, and W.P.M.H. Heemels, "Controllability of a class of bimodal discrete-time piecewise linear systems," Systems and Control Letters, submitted.

[14] W.P.M.H. Heemels and M.K. Camlibel, "Null controllability of discrete-time linear systems with input and state constraints," in 47th IEEE Conference on Decision and Control, 2008, pp. 3487-3492.

[15] H.L. Trentelman, A.A. Stoorvogel, and M. Hautus, Control Theory for Linear Systems, ser. Communications and Control Engineering Series. Springer, 2002.

[16] W.M. Wonham, Linear Multivariable Control: A Geometric Approach. Springer, 3rd edition, 1985. 
[17] G.B. Basile and G. Marro, Controlled and Conditioned Invariants in Linear System Theory. Englewood Cliffs, NJ: Prentice Hall, 1992.

[18] M.K. Camlibel, W.P.M.H. Heemels, and J.M. Schumacher, "On the controllability of bimodal piecewise linear systems," in Hybrid Systems; Computation and Control, ser. Lecture Notes in Computer Science. Springer Berlin / Heidelberg, 2004, vol. 2993, pp. 571614. 